\title{
The Existence of Constant Mean Curvature Foliations of Gowdy 3-Torus Spacetimes *
}

\author{
James Isenberg ${ }^{1}$ and Vincent Moncrief ${ }^{2}$ \\ 1 Department of Mathematics, University of California, Berkeley, CA 94720, USA \\ 2 Department of Physics, Yale University, New Haven, CT 06511, USA
}

\begin{abstract}
We consider the class of smooth, maximally extended, globally hyperbolic, vacuum, Gowdy spacetimes on $T^{3} \times R$ and prove that these spacetimes are globally foliated by space-like, constant mean curvature hypersurfaces. Our results can easily be extended to cover electrovac solutions of the same symmetry type and can probably be extended to cover other spacetime topologies as well.
\end{abstract}

\section{Introduction}

The importance of constant mean curvature (CMC) hypersurfaces as useful tools for studying the physics of general relativistic spacetimes is widely recognized. Nevertheless little is yet known about the class of spacetimes which admit them. It has been conjectured that every maximally extended, globally hyperbolic, spatially compact solution of Einstein's equations (in vacuum or with "reasonable" source field coupling) can be foliated by CMC hypersurfaces [1,2]. However this conjecture is known to be true only for a handful of examples such as the spatially homogeneous cosmological models [3]. In this paper we shall extend the validity of the conjecture to a rather wide class of spacetimes - the Gowdy models [4] with $T^{3} \times R$ topology. For simplicity we consider only vacuum spacetimes. However, one could easily extend our result to Einstein-Maxwell spacetimes of the same symmetry type.

This paper is not intended to provide a review of the known results concerning existence and uniqueness of CMC hypersurfaces; nor does it discuss the usefulness of such hypersurfaces once they have been obtained. (For the former we refer the reader to the article by Marsden and Tipler [5]; for the latter we suggest the 1979 Battelle conference proceedings edited by Smarr [6]). Rather, the paper is aimed directly at proving our result. We proceed by first defining the Gowdy metrics and then stating and proving our theorem. We conclude with a discussion of the possible

* Research supported in part by NSF grant No. PHY79-16482 at Yale and No. PHY79-13146 at Berkeley 
usefulness of the methods developed here for studying CMC foliations of other spacetimes.

\section{Gowdy $T^{3}$ Spacetimes}

Definition. $(M, g)$ is a Gowdy $T^{3}$ vacuum spacetime if

a) $M=T^{3} \times R^{+}, R^{+}=(0,+\infty)$.

b) In suitable coordinates $\left\{\left(t, \theta, x^{a}\right) \mid t \in R^{+}, \theta \in[0,2 \pi), x^{a} \in[0,2 \pi)\right\}$, where $\theta$ and $\left\{x^{a}\right\}=\left\{x^{1}, x^{2}\right\}$ are periodic coordinates on $T^{3}, g$ takes the form

$$
g=\exp (2 \omega)\left(-d t^{2}+d \theta^{2}\right)+g_{a b} d x^{a} d x^{b}, a, b \in[1,2],
$$

where $\omega$ is a smooth function of $(t, \theta)$ and $g_{a b}$ is, for each $(t, \theta)$, a Riemannian metric over $T^{2}$ whose components are smooth functions of $(t, \theta)$ and $\left(\operatorname{det} g_{a b}\right)^{1 / 2}=t$.

c) $\omega$ and $g_{a b}$ are chosen so that $g$ satisfies the vacuum Einstein equations $[4,7]$.

Each of the spacetimes admits a pair of commuting, spacelike, non-vanishing Killing vector fields $\partial / \partial x^{1}$ and $\partial / \partial x^{2}$. Indeed, almost all vacuum spacetimes on $T^{3} \times R^{+}$ characterized by such an isometry group (spacelike, $T^{2}$ ) satisfy the above definition and therefore fit the Gowdy class (the extra restrictions are described by Gowdy [8]).

Recent work [9] has clarified the global properties of the Gowdy $T^{3}$ spacetimes. In particular, it has been shown that the maximally extended globally hyperbolic region of any of these spacetimes can be completely foliated by the $t=$ constant Cauchy surfaces $\Sigma_{t}$, where $t=\left(\operatorname{det} g_{a b}\right)^{1 / 2}$ and $t$ ranges over $(0,+\infty)^{1}$.

Each of these surfaces $\Sigma_{t}$ has a smooth induced Riemannian metric

$$
\gamma\left(\Sigma_{t}\right)=\exp (2 \omega) d \theta^{2}+g_{a b} d x^{a} d x^{b} .
$$

Furthermore one finds that the mean curvature function $\operatorname{tr}\left(K\left(\Sigma_{t}\right)\right)$ blows up uniformly as $t \rightarrow 0^{+}$so that the Gowdy $T^{3}$ spacetimes all have "crushing singularities" in the sense of Eardley and Smarr [2].

To simplify the analysis we shall restrict our attention throughout to $C^{\infty}$ spacetimes. The results of ref. [7] apply to a larger class of solutions and one could extend our theorem accordingly.

\section{Theorem and Proof}

Theorem. Let $(M, g)$ be a maximally extended, globally hyperbolic, vacuum, Gowdy $T^{3}$ spacetime. Then there exists a unique (up to spatial diffeomorphism) spacelike CMC foliation $i_{\lambda}$ of $(M, g)$ which covers $(M, g)$. The mean curvature $\operatorname{tr}(K(\lambda))$ of the surfaces $i_{\lambda}\left(T^{3}\right)$ is a monotonic function of $\lambda$ which approaches $-\infty$ as $i_{\lambda}\left(T^{3}\right)$ approaches the "crushing singularity" of $(M, g)$.

Proof. The basic idea of the proof is as follows. We start by proving two lemmas.

1 This coordinate condition has the property that the hypersurfaces $\Sigma_{t}$ are each foliated by flat two-tori which are integral manifolds of the Killing fields $\partial / \partial x^{a}$ and which have surface area $(2 \pi)^{2} t$. It was introduced by Gowdy to simplify the form of Einstein's equations 
The first lemma shows that if $(M, g)$ admits an "initial" CMC hypersurfaces $\Sigma_{\lambda_{0}}$ then it admits a global foliation $i_{\lambda}$ by CMC hypersurfaces $\Sigma_{\lambda}=i_{\lambda}\left(T^{3}\right)$. This foliation is unique and monotonic. The second lemma shows that Cauchy data for any Gowdy spacetime can be connected by a smooth curve of Gowdy Cauchy data to initial data for a Kasner spacetime. Kasner spacetimes are spatially homogeneous and, as is well known, admit global $\mathrm{CMC}$ foliations for which $\operatorname{tr}(K)$ ranges over $(-\infty, 0)$. To complete the proof of the theorem we use a slightly modified version of a "method of continuity" argument of Marsden and Tipler [5] to show that along the curve of Gowdy spacetimes generated by the curve of Cauchy data just described, one can construct a curve of embeddings of CMC hypersurfaces with a fixed value of $\operatorname{tr}(K)=k_{0}<0$. The estimates of ref. [7] can be used to show that this curve of embeddings cannot degenerate and thus always extends to an embedding for the initially given spacetime $(M, g)$. This provides the initial CMC hypersurfaces needed to construct the global foliation.

We now state formally and prove the two lemmas after which we shall complete the proof of the theorem.

Lemma (1). Let $(M, g)$ be a maximally extended, globally hyperbolic Gowdy $T^{3}$ spacetime and assume that $\Sigma_{\lambda_{0}}$ is a smooth spacelike CMC hypersurface in $(M, g)$ with mean curvature $k_{0}<0$. Then $(M, g)$ admits a unique, monotonic foliation $i_{\lambda}$ by spacelike CMC hypersurfaces $\Sigma_{\lambda}=i_{\lambda}\left(T^{3}\right)$ which covers $(M, g)$. The mean curvature $\operatorname{tr}(K(\lambda))$ of $\Sigma_{\lambda}$ approaches $-\infty$ as $\Sigma_{\lambda}$ approaches the "crushing singularity" of $(M, g)$.

Lemma Proof. We know from the proof of Theorem (3) of Marsden and Tipler $[5,10]$ that there exists a unique, monotonic, local CMC foliation of $(M, g)$ defined near $\Sigma_{\lambda_{0}}$, and that this local foliation can be extended so long as the CMC hypersurfaces $\Sigma_{\lambda}=i_{\lambda}\left(T^{3}\right)$ remain bounded away from the singularity and away from the causal cone.

Suppose we have a local CMC foliation of $(M, g)$ near $\Sigma_{\lambda_{0}}$ contained within an open submanifold $\left(t_{0}, t_{1}\right) \times T^{3}$ of $M$. Here $t_{0}$ and $t_{1}$ are some values of the Gowdy time function $t$ satisfying $0<t_{0}<t_{1}<\infty$. We shall show that this foliation is uniformly spacelike-that the hypersurfaces cannot approach the causal cone unless $t_{0} \rightarrow 0$ or $t_{1} \rightarrow \infty$.

A result of Fischer, Marsden and Moncrief [11] shows that the spacelike Killing fields of $(M, g)$ must be tangent to any CMC hypersurface of $(M, g)$. This also follows as a corollary to the uniqueness of $\operatorname{tr}(K)=$ constant $\neq 0$ hypersurfaces as discussed by Marsden and Tipler [12]. It follows that if $\Sigma_{\lambda}$ is a CMC hypersurface in the local foliation there must exist a smooth function $h_{\lambda}: S^{1} \rightarrow R^{+}$such that $\Sigma_{\lambda}$ is defined in Gowdy coordinates $\left(t, \theta, x^{a}\right)$ by $t=h_{\lambda}(\theta)$. Moreover, since $\Sigma_{\lambda}$ is a CMC hypersurface, $h_{\lambda}$ must satisfy the equation defining a CMC embedding,

$$
-\frac{d}{d \theta}\left[\frac{e^{\omega} h_{\lambda} h_{\lambda}^{\prime}}{\left(1-\left(h_{\lambda}^{\prime}\right)^{2}\right)^{1 / 2}}\right]=\left.h_{\lambda} e^{\omega}\left\{\left(1-\left(h_{\lambda}^{\prime}\right)^{2}\right)^{1 / 2}\left[\frac{\partial \omega}{\partial t}(t, \theta)+\frac{1}{t}\right]+\operatorname{tr}(K) e^{\omega}\right\}\right|_{t=h_{\lambda}(\theta)},
$$

where $h_{\lambda}^{\prime} \equiv d h_{\lambda} / d \theta, \omega=\omega\left(h_{\lambda}(\theta), \theta\right)$ (recall Eq. (2.1) for the definition of $\left.\omega(t, \theta)\right)$ and where $\operatorname{tr}(K)$ is the (constant) mean curvature of the embedded surface. Note that the 
induced metric on $\Sigma_{\lambda}$ is given by

$$
\gamma_{\lambda}=e^{2 \omega}\left(1-\left(h_{\lambda}^{\prime}\right)^{2}\right) d \theta^{2}+g_{a b} d x^{a} d x^{b}
$$

and is strictly spacelike provided $\left|h_{\lambda}^{\prime}\right|<1$.

We want to use Eq. (3.1) to show that $\left|h_{\lambda}^{\prime}\right|$ remains bounded away from unity on the local foliation. To do this we first use Einstein's equations to reexpress $\partial \omega / \partial t$ in terms of the energy density $\mathscr{E}$ defined in $[7] .^{2} \mathscr{E}(t, \theta)$ is expressible in terms of $\left(g_{a b}, \partial g_{a b} / \partial t, \partial g_{a b} / \partial \theta\right)(t, \theta)$ and is a positive definite quadratic form in the derivatives. The Einstein equations give

$$
\omega_{, t}=-\frac{1}{4 t}+\frac{1}{2} \mathscr{E}(t, \theta)
$$

The energy estimates derived in Sect. III of ref. [7] show that $\omega(t, \theta)$ and $\mathscr{E}(t, \theta)$ are smooth functions on $\left[t_{0}, t_{1}\right] \times S^{1}$ for any $\left[t_{0}, t_{1}\right] \subset(0,+\infty)$. Integrating Eq. (3.1) from $\theta_{0}$ to $\theta_{1}$ where $\theta_{1}$ is arbitrary but $\theta_{0}$ is chosen so that $h_{\lambda}^{\prime}\left(\theta_{0}\right)=0$ (such a value of $\theta_{0}$ always exists by the mean value theorem on a circle) we get, after a straightforward sequence of estimates,

$$
\begin{aligned}
& \left.\left|\frac{e^{\omega} h_{\lambda} h_{\lambda}^{\prime}}{\left(1-\left(h_{\lambda}^{\prime}\right)^{2}\right)^{1 / 2}}\right|\right|_{\theta=\theta_{1}} \\
& \quad \leqq\left.\int_{0}^{2 \pi} d \theta\left[h_{\lambda} e^{\omega}\left\{\left(1-\left(h_{\lambda}^{\prime}\right)^{2}\right)^{1 / 2}\left[\frac{1}{2} \mathscr{E}(t, \theta)+\frac{3}{4 t}\right]+e^{\omega}|\operatorname{tr}(K)|\right\}\right]\right|_{t=h_{\lambda}(\theta)} .
\end{aligned}
$$

From the boundedness of $\omega$ and $\mathscr{E}$ and the fact that $h_{\lambda}(\theta) \in\left[t_{0}, t_{1}\right] \subset(0,+\infty)$ we thus find that $\left|h_{\lambda}^{\prime} /\left(1-\left(h_{\lambda}^{\prime}\right)^{2}\right)^{1 / 2}\right|$ is bounded by a finite constant. It follows that $\left|h_{\lambda}^{\prime}\right|$ is bounded away from unity and thus that the CMC hypersurfaces of the local foliation are uniformly spacelike.

Since this argument holds for any $\left[t_{0}, t_{1}\right] \subset(0,+\infty)$ it follows from the continuation argument of Marsden and Tipler that the (unique, monotonic) local foliation $i_{\lambda}$ can always be extended until the "leaves" of the foliation, $\Sigma_{\lambda}=i_{\lambda}\left(T^{3}\right)$, approach the boundaries of the maximal Cauchy development at $t=0$ and $t=+\infty$. We shall now show that the leaves $\Sigma_{\lambda}$ cannot approach the boundaries at $t=0$ and $t=\infty$ without foliating a whole neighbourhood of these boundaries.

First note that since $\left|h_{\lambda}^{\prime}\right|<1$ on any local foliation, the Gowdy time function $t$ has bounded variations on each of the CMC hypersurfaces. More precisely, since $\theta \in[0,2 \pi)$ and $\left|h_{\lambda}^{\prime}\right|<1$ we get

$$
0 \leqq \sup _{t_{\lambda}\left(T^{3}\right)}(t)-\inf _{t_{\lambda}\left(T^{3}\right)}(t) \leqq 2 \pi .
$$

It follows that the hypersurfaces $i_{\lambda}\left(T^{3}\right)$ cannot approach the boundary of $(M, g)$ at $t=+\infty$ without in fact foliating a full neighbourhood of $t=+\infty$.

Finally, consider the region near $t=0$. A result due primarily to Brill and Flaherty $[13,14]$ shows that if there exists a CMC hypersurface $S_{k_{0}}$ with

2 In the notation of ref. [7], $\mathscr{E}=\frac{1}{2} t G_{A B}\left(\dot{X}^{A} \dot{X}^{B}+X^{A^{\prime}} X^{B^{\prime}}\right)$, where $X^{A}$ and $G_{A B}$ are defined by Eqs. (2.1)(2.5), (2.8) and (2.10) of that reference. The integral of $\mathscr{E}$ gives the total "energy" defined by Eq. (3.1) of ref. [7] 
$\operatorname{tr}\left(K\left(S_{k_{0}}\right)\right)=k_{0}<0$ and compact spacelike hypersurfaces $\Sigma_{+}$and $\Sigma_{-}$such that

$$
0<\sup \left(-\operatorname{tr}\left(K\left(\Sigma_{-}\right)\right)\right)<-k_{0}<\inf \left(-\operatorname{tr}\left(K\left(\Sigma_{+}\right)\right)\right)<\infty,
$$

then $S_{k_{0}}$ lies within the compact subset of $M$ bounded by $\Sigma_{+}$and $\Sigma_{-}$. However, we showed in [7] that for any Gowdy spacetime and any $t_{0}>0$ there exist monotonic functions $\sigma_{-}, \sigma_{+}:\left(0, t_{0}\right] \rightarrow(0,+\infty)$ such that, for any $t=$ constant hypersurface $\Sigma_{t}$, with $t \in\left(0, t_{0}\right]$,

$$
\begin{aligned}
& \sup \left(-\operatorname{tr}\left(K\left(\Sigma_{t}\right)\right)\right) \leqq \sigma_{+}(t), \\
& \inf \left(-\operatorname{tr}\left(K\left(\Sigma_{t}\right)\right)\right) \geqq \sigma_{-}(t) .
\end{aligned}
$$

Furthermore $\sigma_{+}$and $\sigma_{-}$increase without bound as $t \rightarrow 0^{+}$. The existence of $\sigma_{+}$ follows from the energy estimates which demonstrate global existence of the Gowdy solutions on $(0,+\infty) \times T^{3}$. We derived an explicit form for $\sigma_{-}$in ref. [7] to show that $\inf \left(-\operatorname{tr}\left(K\left(\Sigma_{t}\right)\right)\right) \rightarrow \infty$ as $t \rightarrow 0^{+}$(i.e., to show that the edge at $t=0^{+}$is a "crushing singularity").

It follows from the above remarks that a CMC foliation $i_{\lambda}$ cannot approach the boundary at $t=0^{+}$unless $-\operatorname{tr}\left(K\left(i_{\lambda}\left(T^{3}\right)\right)\right) \equiv-k_{\lambda}$ increases without bound. However the same remarks show that for any $t \in\left(0, t_{0}\right]$ one can choose $-k_{\lambda}$ sufficiently large that the corresponding CMC hypersurface $S_{\lambda}=i_{\lambda}\left(T^{3}\right)$ lies within $(0, t] \times T^{3}$. Thus the CMC hypersurfaces foliate a whole neighbourhood of the crushing singularity at $t=0^{+}$and the mean curvature function approaches $-\infty$ as $S_{\lambda}$ approaches this boundary.

To prove the second lemma we need some results on the Cauchy problem for Gowdy spacetimes. In [7] we introduced a convenient parameterization for the Riemannian metric $g_{a b}$ (satisfying $(\operatorname{det} g)^{1 / 2}=t$ ) in terms of two unconstrained functions $\left\{X^{A}(t, \theta)\right\}, A=1,2$. From the Lagrangian for their equations of motion one can define conjugate momenta $\left\{P^{A}(t, \theta)\right\}$. The only restriction upon the choice of $\left\{X^{A}, P^{A}\right\}$ on some $t=t_{0}=$ constant initial surface is the constraint,

$$
\left.\int_{S^{1}} d \theta\left\{P^{A} \frac{\partial X^{A}}{\partial \theta}\right\}\right|_{t=t_{0}}=0
$$

which is necessary and sufficient to ensure that $\omega(t, \theta)$, determined by Einstein's equations to be

$$
\omega(t, \theta)=\omega\left(t, \theta_{0}\right)+\frac{1}{2} \int_{\theta_{0}}^{\theta} d \theta^{\prime}\left\{P^{A} \frac{\partial X^{A}}{\partial \theta}\right\}\left(t, \theta^{\prime}\right),
$$

be a smooth function on spacetime.

Lemma (2). Let $\left(M, g_{1}\right)$ be any chosen Gowdy spacetime and let $\left\{X_{(1)}^{A}\right.$, $\left.P_{(1)}^{A}\right\}$ be Cauchy data for $\left(M, g_{1}\right)$ specified at $t=t_{0}$. Then there exists a spatially homogeneous Gowdy spacetime $\left(M, g_{0}\right)$ with Cauchy data $\left.\left\{X_{(0)}^{A}, P_{(0)}^{A}\right\}\right|_{t=t_{0}}$ and a smooth curve of Gowdy Cauchy data $\left.\left\{X_{(s)}^{A}, P_{(s)}^{A}\right\}\right|_{t=t_{0}}$, with $s \in[0,1]$, connecting $\left\{X_{(0)}^{A}, P_{(0)}^{A}\right\}$ and $\left\{X_{(1)}^{A}, P_{(1)}^{A}\right\}$. The corresponding one parameter family of Gowdy spacetimes $\left(M, g_{s}\right)$ is smooth if restricted to a compact subset $\left[t_{a}, t_{b}\right] \times T^{3} \subset M$. The spatially homogeneous solution is globally foliated by CMC hypersurfaces (which 
coincide with the $t=$ constant surfaces) and the mean curvature, $\operatorname{tr}(K)$, of these surfaces ranges over $(-\infty, 0)$.

Lemma Proof. For this argument any spatially homogeneous Gowdy solution would suffice for $\left(M, g_{0}\right)$ since all such "Kasner spacetimes" have the properties mentioned in the lemma. For definiteness however we shall choose $g_{0}$ to be the "flatKasner" metric given by [15]

$$
g_{0}=-d t^{2}+d \theta^{2}+t^{2}\left(d x^{1}\right)^{2}+\left(d x^{2}\right)^{2} .
$$

One easily verifies that the $t=$ constant surfaces define a CMC foliation with $\operatorname{tr}(K)$ ranging over $(-\infty, 0)$.

To construct the desired curve of Gowdy Cauchy data we set, for $s \in[0,1]$,

$$
\begin{aligned}
X_{(s)}^{A} & =(1-s) X_{(0)}^{A}+s X_{(1)}^{A}, \\
P_{(s)}^{A} & =(1-s) P_{(0)}^{A}+s P_{(1)}^{A} .
\end{aligned}
$$

Noting that the Kasner data satisfies $\partial X_{(0)}^{A} / \partial \theta=\partial P_{(0)}^{A} / \partial \theta=0$, it is straightforward to verify that condition (3.8) is satisfied everywhere along the curve. One can complete the choice of initial data for $\left(M, g_{s}\right)$ by choosing a smoothly varying constant $\omega_{s}\left(\theta_{0}, t_{0}\right)$ and definining $\omega_{s}\left(\theta, t_{0}\right)$ through Eq. (3.9).

The global existence theorem of ref. [7] assures the smooth dependence of the solutions $\left(M, g_{s}\right)$ upon their Cauchy data in the sense that on compact subsets, $\left[t_{a}, t_{b}\right] \times T^{3}$, of $M$ the curve of metrices $g_{s}$ is smooth in a suitable Sobolev topology. One can choose the Sobolev spaces in such a way that the metric components, $g_{\mu v}(t, \theta, s)$, are $C^{k}$ functions of $(t, \theta, s)$ for any $k \geqq 2$.

To complete the proof of the main theorem we use the "method of continuity" technique to show that, for a suitably chosen constant $k_{0}$, one can construct a curve of embeddings $i_{s}$ of $T^{3}$ into $M$ such that $i_{s}\left(T^{3}\right)$ is a $\operatorname{tr}(K)=k_{0}$, spacelike hypersurface of $\left(M, g_{s}\right)$. One can appeal to a (slightly modified[16]) result of Marsden and Tipler (Theorem (4) of ref. [5]) to show that such a curve of embeddings always exists on some neighbourhood of the Kasner solution $\left(M, g_{0}\right)$ and that the curve can always be extended until $i_{s}\left(T^{3}\right)$ approaches the singularity of $\left(M, g_{s}\right)$ or turns null. We shall exclude these possibilities by arguments similar to those of Lemma (1).

To choose a suitable value for $k_{0}$ let $\Gamma:[0,1] \times S^{1} \rightarrow R$ be defined by $\Gamma(s, \theta)=$ $-\operatorname{tr}\left(K_{s}\left(t_{0}, \theta\right)\right)$, where $\operatorname{tr}\left(K_{s}\right)$ is the mean curvature function for $g_{s}$. Now $\Gamma$ is continuous on the compact set $[0,1] \times S^{1}$ and thus achieves some maximum value $\Gamma_{\max }$. Choose $k_{0}$ such that $\Gamma_{\max }<-k_{0}<\infty$. This will ensure that if a $\operatorname{tr}\left(K_{s}\right)=k_{0}$ surface exists for $\left(M, g_{s}\right)$ it will lie within the subset $\left(0, t_{0}\right] \times T^{3}$ and thus remain bounded away from the edge at $t=\infty$.

The derivation of the second inequality in Eq. (3.7), given in Sect. III $C$ of ref. [7], shows that $\sigma_{-}$can be expressed as $\sigma_{-}(t)=C / t^{3 / 4}$ where $C$ is a constant (for each solution) which varies smoothly with the initial data. Thus one can always choose a $t_{1}$ such that $0<t_{1}<t_{0}$ and

$$
\begin{gathered}
-\operatorname{tr}\left(K_{s}\left(\Sigma_{t}\right)\right) \geqq-k_{0}>0, \\
\forall t \in\left(0, t_{1}\right) \quad \text { and } \quad \forall s \in[0,1] .
\end{gathered}
$$


It follows from the proof of Lemma (1) that if $\left(M, g_{s}\right)$ admits a $\operatorname{tr}\left(K_{s}\right)=k_{O}$ hypersurface, then this surface lies within the compact subset $\left[t_{1}, t_{0}\right] \times T^{3}$ of $M$. Thus as one varies $s$ the embedding $i_{s}\left(T^{3}\right)$ remains bounded away from the edge of $\left(M, g_{s}\right)$ at $t=0$.

For $s$ sufficiently small we therefore have a continuous curve of $\operatorname{tr}\left(K_{s}\right)=k_{0}$ embeddings defined, in Gowdy coordinates, by $t_{s}=h_{s}(\theta)$. Here $h_{s}(\theta)=h(s, \theta)$ for some continuous function $h:\left[0, s_{0}\right] \times S^{1} \rightarrow\left[t_{1}, t_{0}\right]$ and either $s_{0}=1$ or else the embedded surfaces approach the null cones of $\left(M, g_{s}\right)$ as $s \rightarrow s_{0}$. When evaluated for $g_{s}$, however, the energy density $\mathscr{E}$ and metric function $\omega$ become continuous functions of $(s, t, \theta)$ on the compact set $[0,1] \times\left[t_{1}, t_{0}\right] \times S^{1}$. Therefore, one can repeat the argument leading from Eq. (3.1) to Eq. (3.4), making the obvious modifications $h_{\lambda} \rightarrow h_{s}$, etc., to show that $\left|h_{s}^{\prime}\right|$ remains bounded away from unity as $s \rightarrow s_{0}$. It follows that the embedded hypersurfaces remain bounded away from the null cones for all $s_{0} \leqq 1$.

Thus the curve of embeddings $i_{s}\left(T^{3}\right)$ cannot degenerate as $s \rightarrow 1$ and so, by the continuity argument of Marsden and Tipler, extends to define a spacelike CMC embedding for $\left(M, g_{1}\right)$. Since $\left(M, g_{1}\right)$ was an arbitrary Gowdy spacetime, this construction completes the proof of the main theorem.

It is worth noting that our proof does not show that $\operatorname{tr}\left(K\left(i_{\lambda}\left(T^{3}\right)\right)\right) \rightarrow 0$ as $i_{\lambda}\left(T^{3}\right)$ approaches the edge of $(M, g)$ at $t=+\infty$. We believe this is always true but could not find a simple proof. To prove this conjecture it would suffice to show that $\sup \left(-\operatorname{tr}\left(K\left(\Sigma_{t}\right)\right)\right) \rightarrow 0$ as $t \rightarrow+\infty$ (i.e., to show that $\operatorname{tr}(K)$ tends uniformly to zero in the Gowdy chart). One can probably prove this with a slight extension of the arguments given in ref. [7].

\section{Conclusion}

There are several straightforward extensions one can make of the results derived here. First, one can include the coupling to Maxwell fields of the same symmetry type. The needed global existence theorem for the coupled Einstein-Maxwell equations (with Gowdy symmetry and $T^{3} \times R$ topology) has been given elsewhere [17]. The remainder of the argument is essentially the same as that given here.

In addition one can study spacetimes with different topologies. Gowdy considered three different spatial topologies, the three-torus, the three-sphere and the "wormhole," $S^{2} \times S^{1}$. To prove a corresponding global existence theorem for the $S^{3}$ and $S^{2} \times S^{1}$ topologies is not completely straightforward however since, in these cases, the orbits of one or both Killing fields degenerate at certain "axes of symmetry" within the spacetimes. In adapting the coordinates to the symmetry (which is important for making the global existence estimates) one introduces coordinate singularities at the symmetry axes and renders the task of making estimates more difficult. We believe that this is merely a "technical problem" which one could overcome with additional effort but we have not carried this effort out.

On the other hand, however, there is an infinite dimensional sub-family of the Gowdy spacetimes which one can compute almost explicitly for each of the three topological types. These are the "polarized" solutions which have orthogonal 
Killing fields and which obey separable, linear hyperbolic equations [18]. For these solutions one does not need a global existence theorem since the global behavior (e.g., the occurrence of crushing singular boundaries of the maximal Cauchy developments) can be studied explicitly. We believe that a slightly modified version of our argument could be used to prove the existence of global CMC foliations for these polarized Gowdy spacetimes.

Finally one can study spacetimes of lower symmetry. In a recent paper[19] one of us has shown how to construct an infinite dimensional family of analytic vacuum spacetimes on $T^{3} \times R$, each having only one Killing vector field and each having a smooth Cauchy horizon and an extension of Taub-NUT type[20]. The construction shows that the Cauchy horizons in these solutions are always "crushing singularities" in the Eardley-Smarr sense [2]. The main problem in extending our results to this family of spacetimes is proving that the CMC hypersurfaces of a local foliation cannot turn null. We believe that this can probably be shown by a generalization of the argument in Lemma (1). A study of this question as well as the associated global existence problem for cosmological spacetimes with one Killing field is currently in progress.

Acknowledgement. We thank the Aspen Center for Physics for providing a stimulating and beautiful place in which to carry out this work.

\section{References}

1. For a discussion of this conjecture and its relevance to the cosmic censorship conjecture see Moncrief, V., Eardley, D.: Gen. Rel. Grav. 13, 887 (1981)

2. Eardley D., Smarr, L.: Phys. Rev. D19, 2239 (1979). This paper discusses primarily the analogous conjecture in the non-compact case and introduces the notion of "crushing singularities" which will play an important role in the present paper

3. The hypersurfaces of transitivity of the isometry group automatically have constant mean curvature in such models. This result is independent of Einstein's equations

4. Gowdy, R.: Ann. Phys. (N.Y.) 83, 203 (1974). See also Gowdy, R.: Phys. Rev. Lett. 27, 826 and 1102 (E) (1971)

5. Marsden, J., Tipler, F.: Phys. Rep. 66, 109 (1980)

6. Sources of gravitational radiation. Smarr, L.,(ed.) Cambridge: Cambridge Press, 1979; see especially the articles by York, J., Eardley, D., Smarr, L., Wilson, J.

7. Moncrief, V.: Ann. Phys. (N.Y.) 132, 87 (1980)

8. The extra restrictions involve the alignment of the principal axes of expansion of the spacetime with the axes of toroidal identification. See the first paper of [4] for a more complete discussion

9. The basic global existence theorem referred to in this paper is proven in [7] where it is also shown that the Gowdy models always have crushing singularities in the sense of Eardley and Smarr (c.f. [2])

10. The Marsden-Tipler arguments require some slight modifications to be applicable to the spacetimes considered here. Their requirement that $(M, g)$ be non-flat can be replaced by the requirement that $(M, g)$ admit no global timelike Killing field. In particular, the flat Kasner solution used below in this paper need not be excluded. Furthermore, one can replace the phrase "maximal hypersurface" by "constant mean curvature hypersurface" in Marsden and Tipler's argument and prove the analogous theorem using the same methods. The modified version of their Theorem (3) does not lead to "Wheeler universes" but still implies the existence of global CMC foliations

11. Fischer, A., Marsden, J., Moncrief, V.: Ann. Inst. H. Poincaré, 33, 147 (1980). See Proposition 2.3 and the discussion immediately following. The condition $\pi_{0} \neq 0$ or $g_{0}$ is not flat excludes precisely those spacetimes which have a timelike Killing field. No Gowdy metric has such a Killing field. 
12. See Corollary (2) on p. 121 of [5]

13. Brill, D., Flaherty, F.: Commun. Math. Phys. 50, 157 (1976). For the vacuum case see [14]

14. See especially the discussion on pp. $119-120$ of [5]

15. See the remarks regarding flat spacetimes in [10]

16. The continuity argument associated with Theorem (4) of [5] can easily be modified to cover the spacetimes studied here. The needed modifications are essentially the same as those discussed in [10]. The modified theorem implies the existence of a CMC hypersurface instead of a maximal one

17. Moncrief, V.: Lagrangian submanifolds of extendible spacetimes. (in preparation)

18. See the second paper of [4]

19. Moncrief, V.: Neighborhoods of Cauchy horizons in cosmological spacetimes with one killing field. Ann. Phys. (N.Y) 141, 83 (1982).

20. For a nice discussion of the properties of Taub-NUT spacetime see Hawking, S.W., Ellis, G.F.R.. The large scale structure of spacetime. Cambridge: Cambridge Press 1973

Communicated by S.-T. Yau

Received February 1, 1982; in revised form May 3, 1982 
